\title{
Revisiting Unrestricted Rebut and Preferences in Structured Argumentation. *
}

\author{
Jesse Heyninck and Christian Straßer \\ Ruhr University Bochum, Germany \\ jesse.heyninck@rub.de, christian.strasser@rub.de
}

\begin{abstract}
In structured argumentation frameworks such as $\mathrm{ASPIC}^{+}$, rebuts are only allowed in conclusions produced by defeasible rules. This has been criticized as counter-intuitive especially in dialectical contexts. In this paper we show that $\mathrm{ASPIC}^{-}$, a system allowing for unrestricted rebuts, suffers from contamination problems. We remedy this shortcoming by generalizing the attack rule of unrestricted rebut. Our resulting system satisfies the usual rationality postulates for prioritized rule bases.
\end{abstract}

\section{Introduction}

Structured argumentation offers a formal method for defeasible reasoning. Many approaches distinguish between strict and defeasible inference rules. Unlike strict rules, a defeasible rule warrants the truth of its conclusion only provisionally: it is retracted in case good counter-arguments are encountered. Different types of argumentative attacks can be distinguished. E.g., if an argument $a$ concludes the contrary of an argument $b$ it is said to rebut $b$. In many formalisms, such as ASPIC ${ }^{+}$[Modgil and Prakken, 2013; 2014], rebut is restricted in such a way that an argument $b$ cannot be rebutted if its conclusion is obtained by a strict rule. In [Caminada et al., 2014], it has been argued that especially in dialectical contexts unrestricted rebut is more natural. As a consequence, the system ASPIC $^{-}$has been proposed in which rebut is unrestricted.

In [Caminada and Amgoud, 2007] several rationality postulates have been proposed. E.g., the output of an argumentation system should be consistent and closed under the strict rules. For multi-extension semantics (e.g., preferred and stable semantics), $\mathrm{ASPIC}^{-}$violates these postulates unlike ASPIC $^{+}$(given some restrictions) [Prakken, 2011]. Thus, unrestricted rebut can lead to undesired behaviour and one has to be careful when devising systems utilizing it. The situation is different for the single-extension grounded semantics where both $\mathrm{ASPIC}^{+}$and $\mathrm{ASPIC}^{-}$are well-behaved relative to the postulates in [Caminada and Amgoud, 2007].

\footnotetext{
${ }^{*}$ We thank M. Beirlaen, M. Caminada and the anonymous reviewers for helpful comments. Our research is supported by the Alexander von Humboldt-Foundation.
}

However, as shown in this article, ASPIC $^{-}$does not satisfy other standards such as Crash-Resistance and NonInterference. Ideally, a reasoning system should not lose consequences if irrelevant information is added to the knowledge base. As demonstrated in [Wu, 2012] for ASPIC, the lack of Crash-Resistance and Non-Interference is especially threatening if the underlying strict rules are domain-independent. This is typically the case if the strict rules are induced by an underlying logic such as classical logic (in short, CL). Given an inconsistent knowledge base and strict rules such as logical explosion, for any formula an argument with a contrary conclusion can be constructed.

So far, there are not many results that establish CrashResistance and Non-Interference for systems in the ASPICfamily. An exception is [Wu, 2012] where Crash-Resistance is established for ASPIC Lite, where priorities over defeasible rules are not taken into account. In [Grooters and Prakken, 2016] a system with restricted rebut is introduced that avoids logical explosion by using a sub-classical logic as a base logic. For any completeness-based semantics a weakened version of Crash-resistance and Closure are shown for total pre-orders expressing priority relations between the defeasible rules. For multi-extension semantics a counter-example for full Crash-Resistance is provided.

For approaches with unrestricted rebut, to the best of our knowledge, there have been no investigations into CrashResistance or Irrelevance. This paper therefore answers the following pertinent question: is it possible to define a framework for structured argumentation that gives a sensible output when the strict rules are domain-independent? We will answer this question positively by defining the system ASPIC ${ }^{\ominus}$. We consider total pre-orders expressing priority relations between the defeasible rules. To the best of our knowledge, this is the first such result for frameworks in the ASPIC-family.

\section{The ASPIC-family}

A well-known, general and popular family of frameworks for structured argumentation is the ASPIC-family. In ASPIC arguments are constructed using an argumentation system. ${ }^{1}$

\footnotetext{
${ }^{1}$ In this paper we will, due to spatial restrictions, omit several features of the original ASPIC ${ }^{+}$framework of [Prakken, 2011], such as defeasible premises, issues, undercutting and undermining attacks.
} 
Definition 1. An Argumentation System (AS) is a tuple $A S=\left(\mathcal{L}, \mathcal{S}, \mathcal{D}, \mathcal{K},^{-}, \leqslant\right)$consisting of:

- a formal language $\mathcal{L}$

- a set of strict rules $\mathcal{S} \subseteq 2^{\mathcal{L}} \times \mathcal{L}$ of the form $A_{1}, \ldots, A_{n} \rightarrow$ $B$

- a set of defeasible rules $\mathcal{D} \subseteq 2^{\mathcal{L}} \times \mathcal{L}$ of the form $A_{1}, \ldots, A_{n} \Rightarrow B$.

- an $\mathcal{S}$-consistent ${ }^{2}$ set of strict premises $\mathcal{K} \subseteq \mathcal{L}$.

- a contrariness function ${ }^{-}$from $\mathcal{L}$ to $\mathcal{L} .^{3}$

- a preorder $\leqslant$ over $\mathcal{D}$.

$A_{1}, \ldots, A_{n}$ are called the antecedents and $B$ is called the consequent of $A_{1}, \ldots, A_{n} \rightarrow B$ resp. $A_{1}, \ldots, A_{n} \Rightarrow B$.

Definition 2. Let $A S=(\mathcal{L}, \mathcal{S}, \mathcal{D}, \mathcal{K},-\leqslant)$ be an argumentation system. An argument a is one of the following:

- $a=\langle A\rangle$ where $A \in \mathcal{K}$

$\operatorname{conc}(a)=A, \operatorname{Sub}(a)=\{a\}, \operatorname{DefR}(a)=\emptyset$

- $a=\left\langle a_{1}, \ldots, a_{n} \rightarrow B\right\rangle$ where $a_{1}, \ldots a_{n}$ (with $n \geqslant 0)$ are arguments s.t. there is a strict rule $\operatorname{conc}\left(a_{1}\right), \ldots \operatorname{conc}\left(a_{n}\right) \rightarrow B \in \mathcal{S}$

$\operatorname{conc}(a)=B, \operatorname{Sub}(a)=\{a\} \cup \bigcup_{i=1}^{n} \operatorname{Sub}\left(a_{i}\right)$, and $\operatorname{DefR}(a)=\bigcup_{i=1}^{n} \operatorname{DefR}\left(a_{i}\right)$.

- $a=\left\langle a_{1}, \ldots, a_{n} \Rightarrow B\right\rangle$ where $a_{1}, \ldots a_{n}$ (with $n \geqslant 0)$ are arguments s.t. there is a defeasible rule $\operatorname{conc}\left(a_{1}\right), \ldots \operatorname{conc}\left(a_{n}\right) \Rightarrow B \in \mathcal{D}$

$\operatorname{conc}(a)=B, \operatorname{Sub}(a)=\{a\} \cup \bigcup_{i=1}^{n} \operatorname{Sub}\left(a_{i}\right), \operatorname{DefR}(a)=$ $\left\{\operatorname{conc}\left(a_{1}\right), \ldots \operatorname{conc}\left(a_{n}\right) \Rightarrow B\right\} \cup \bigcup_{i=1}^{n} \operatorname{DefR}\left(a_{i}\right)$.

By $\operatorname{Arg}(A S)$ we denote the set of arguments that can be built from $A S$. An argument $a$ will be called defeasible if $\operatorname{DefR}(a) \neq \emptyset$ and strict otherwise. We lift DefR to sets of arguments as usual: $\operatorname{DefR}\left(\left\{a_{1}, \ldots, a_{n}\right\}\right)=\bigcup_{i=1}^{n} \operatorname{DefR}\left(a_{i}\right)$.

Example 1. The paradigmatic example for generating a set of strict rules $\mathcal{S}_{\mathbf{C L}}$ by an underlying logic is to use CL: $A_{1}, \ldots, A_{n} \rightarrow A \in \mathcal{S}_{\mathbf{C L}}$ iff $\left\{A_{1}, \ldots, A_{n}\right\} \vdash_{\mathbf{C L}} A$. Contrariness is defined by $\bar{A}=\neg A$. We will use this system as a guiding example throughout this paper.

Let $A S_{1}=\left(\mathcal{L}, \mathcal{S}_{\mathbf{C L}}, \mathcal{D}_{1}=\left\{\top \Rightarrow_{2} \neg p \vee \neg q, \top \Rightarrow_{1}\right.\right.$ $\left.\left.p, p \Rightarrow_{1} q\right\}, \emptyset,^{-}, \leqslant\right)$. In this and the following examples, the natural numbers in the subscripts of $\Rightarrow$ are used to express the priority ordering over $\mathcal{D}$, i.e. $\left(A_{1}, \ldots, A_{n} \Rightarrow_{i} B\right) \leqslant$ $\left(A_{1}^{\prime}, \ldots, A_{m}^{\prime} \Rightarrow_{j} B^{\prime}\right)$ iff $i \leqslant j$. Here are some arguments in $\operatorname{Arg}\left(A S_{1}\right)$ :

$$
\begin{array}{llll}
a_{1}: & \top \Rightarrow_{2} \neg p \vee \neg q & a_{2}: & \top \Rightarrow_{1} p \\
a_{3}: & a_{2} \Rightarrow_{1} q & a_{4}: & a_{1}, a_{2} \rightarrow \neg q \\
a_{5}: & a_{2}, a_{3} \rightarrow p \wedge q & a_{6}: & a_{1}, a_{3} \rightarrow \neg p
\end{array}
$$

${ }^{2} \mathcal{K}$ is $\mathcal{S}$-inconsistent iff there is a derivation from $\mathcal{K}$ using $\mathcal{S}$ for $A$ and $\bar{A}$ (for some $A \in \mathcal{L}$ ). $\mathcal{K}$ is $\mathcal{S}$-consistent if it is not $\mathcal{S}$ inconsistent.

${ }^{3}$ In the context of ASPIC $^{+}$usually ${ }^{-}$associates formulas with a set of contrary formulas. To simplify the presentation we opt here for the simpler variant where each formula is associated with a unique contrary formula.

\subsection{Attacks and Defeats}

Definition 3. Where $a, b \in \operatorname{Arg}(A S)$, $a$ unrestrictedly rebuts $b$ (in symbols: $a \mathrm{UrRe} b$ ) iff $\operatorname{conc}(a)=\overline{\operatorname{conc}(b)}$ and $b$ is defeasible.

Definition 4. Where $a, b \in \operatorname{Arg}(A S)$, $a$ restrictedly rebuts (in symbols: $a \operatorname{ReRe} b) b=\left\langle b_{1}, \ldots, b_{n} \Rightarrow B\right\rangle$ iff $\operatorname{conc}(a)=\bar{B}$.

It is clear that for any arguments $a$ and $b$, if $a \operatorname{ReRe} b$ then $a \mathrm{UrRe} b$. The other direction does not hold in general, as witnessed by the fact that in Example 1, where $a_{2}^{\prime}=a_{2} \rightarrow \neg \neg p$, we have $a_{2} \operatorname{UrRe} a_{6}$ but not $a_{2} \operatorname{ReRe} a_{6}$.

When two arguments conflict, one of the arguments may defeat the other due to its higher priority. To account for defeat via priorities, we lift our order $\leqslant$ on $\mathcal{D}$ to an order on arguments via the weakest link lifting(see e.g. [Modgil and Prakken, 2013]): where $a, b \in \operatorname{Arg}(A S), a \preceq b$ iff $\operatorname{DefR}(b)=\emptyset$ or $\exists \alpha \in \operatorname{DefR}(a)$ s.t. $\forall \beta \in \operatorname{DefR}(b): \alpha \leqslant \beta$.

Definition 5. Where $a, b \in \operatorname{Arg}(A S)$ and Att $\in$ $\{$ ReRe, UrRe $\}: a$ defeats $b$ iff there is $a c \in \operatorname{Sub}(b)$ s.t. $a$ Att $c$ and $c \preceq a$. We write $(a, b) \in \operatorname{Att} \preceq(\operatorname{Arg}(A S))$.

Where $\operatorname{Arg}(A S)$ is clear from the context, we will often just write $\mathrm{Att}_{\preceq}$ instead of $\operatorname{Att}_{\preceq}(\operatorname{Arg}(A S))$.

\subsection{Grounded Semantics}

Definition 6. Where Att $\in\{\operatorname{ReRe}, \mathrm{UrRe}\}$, an argumentation framework $(A F)$ for an argumentation theory $A S$ is the pair $(\operatorname{Arg}(A S), \operatorname{Att} \preceq(\operatorname{Arg}(A S)))$.

Given an AF, we can apply Dung's acceptability semantics [Dung, 1995] for evaluating arguments.

Definition 7. Let $A F=(\operatorname{Arg}(A S)$, Att $\preceq)$ be an $A F, \mathcal{A} \subseteq$ $\operatorname{Arg}(A S)$ and $a \in \operatorname{Arg}(A S)$. a is acceptable w.r.t. $\mathcal{A}($ or, $\overline{\mathcal{A}}$ defends a) iff for all $b$ s.t. $(b, a) \in \mathrm{Att} \preceq$ there is $a c \in \mathcal{A}$ s.t. $(c, b) \in \operatorname{Att}_{\preceq}$. We write $\operatorname{Acc}(\mathcal{A})$ for the set of all acceptable arguments w.r.t. $\mathcal{A}$. $\mathcal{A}$ is conflict-free iff there are no $a, b \in \mathcal{A}$ s.t. $(a, b) \in \mathrm{Att} \preceq$. $\mathcal{A}$ is a complete extension iff it is conflictfree and $\operatorname{Acc}(\mathcal{A})=\mathcal{A}$. The minimal complete extension is the grounded extension, written $\mathcal{G}(A F)$.

Remark 1. The grounded extension has the following fixed point characterization: $\mathcal{G}(A F)=\bigcup_{i \geq 0} \mathcal{G}_{i}$ where $\mathcal{G}_{0}=$ $\operatorname{Acc}(\emptyset)$ and $\mathcal{G}_{i+1}=\operatorname{Acc}\left(\mathcal{G}_{i}\right)(i \geq 0)$.

We define a consequence relation based on the grounded extension for AFs as follows.

Definition 8. Where $A F=(\operatorname{Arg}(A S)$, Att $\preceq)$ is an $A F$ for $A S$. $A S \sim^{\text {Att }} \preceq A$ iff there is an argument $a \in \mathcal{G}(A F)$ with $\operatorname{conc}(a)=A$.

\section{Rationality Postulates}

In [Caminada and Amgoud, 2007; Caminada et al., 2011] desirable properties for argumentation-based consequence relations $\sim$ are defined:

Postulate 1. $\sim$ satisfies Direct Consistency if for no argumentation system $A S, A S \sim A$ and $A S \sim \bar{A}$ (for some $A \in \mathcal{L})$. 
Postulate 2. $\sim$ satisfies Closure if for every argumentation system $A S$, if $A S \sim A_{i}$ for $1 \leqslant i \leqslant n$ and $B$ follows via the strict rules of AS from $\left\{A_{1}, \ldots, A_{n}\right\}$ then $A S \sim B$.

Postulate 3. $\sim$ satisfies Indirect Consistency if for no argumentation system $A S$ with strict rules $\mathcal{S}$, there are $A_{1}, \ldots, A_{n}$ s.t. $A S \sim A_{i}$ for $1 \leqslant i \leqslant n$ and $\left\{A_{1}, \ldots, A_{n}\right\}$ is $\mathcal{S}$-inconsistent.

Where $\Delta \subseteq \mathcal{L}$, let $\operatorname{Atoms}(\Delta)$ be the set of all atoms occurring in $\Delta$.

Postulate 4. $\sim$ satisfies Non-Interference if for any two argumentation systems $A S=\left(\mathcal{L}, \mathcal{S}, \mathcal{D}, \mathcal{K},^{-}, \leqslant\right)$and $A S^{\prime}=\left(\mathcal{L}, \mathcal{S}, \mathcal{D}^{\prime}, \mathcal{K}^{\prime},{ }^{-}, \leqslant^{\prime}\right)$, where $\mathcal{K} \cup \mathcal{K}^{\prime}$ is $\mathcal{S}$-consistent, $(\operatorname{Atoms}(\mathcal{D}) \cup \operatorname{Atoms}(\mathcal{K})) \cap\left(\operatorname{Atoms}\left(\mathcal{D}^{\prime}\right) \cup \operatorname{Atoms}\left(\mathcal{K}^{\prime}\right)\right)=\emptyset$, we have: $A S^{\prime} \sim A$ iff $A S^{+} \sim A$ where $A S^{+}=(\mathcal{L}, \mathcal{S}, \mathcal{D} \cup$ $\left.\mathcal{D}^{\prime}, \mathcal{K} \cup \mathcal{K}^{\prime},{ }^{-}, \leq^{+}\right)$and $\operatorname{Atoms}(A) \subseteq \operatorname{Atoms}\left(A S^{\prime}\right)$ (where $\leq^{+}$is such that $\leq\left[\leq^{\prime}\right]$ is the restriction of $\leq^{+}$to $\mathcal{D} \times \mathcal{D}$ $\left.\left[\mathcal{D}^{\prime} \times \mathcal{D}^{\prime}\right]\right)^{4}$

\section{ASPIC $^{-}$, Non-Interference, and Closure: a Dilemma}

In [Caminada et al., 2014] it is shown that $\sim^{\text {UrRe }}$, the consequence relation associated with $\mathrm{ASPIC}^{-}$under the grounded semantics, satisfies Closure and both Consistency postulates. However, if the strict rules are defined on the basis of $\mathbf{C L}$ (see Ex. 1) there are counterexamples to Non-Interference.

Example 2. Let $A S_{2}=\left(\mathcal{L}, \mathcal{S}_{\mathrm{CL}}, \mathcal{D}_{2}=\{\top \Rightarrow p\}, \emptyset,{ }^{-}, \mathcal{D}_{2} \times\right.$ $\left.\mathcal{D}_{2}\right)$ and $A S_{3}=\left(\mathcal{L}, \mathcal{S}_{\mathbf{C L}}, \mathcal{D}_{3}=\{\top \Rightarrow p, \top \Rightarrow s, \top \Rightarrow\right.$ $\left.\neg s\}, \emptyset,{ }^{-}, \mathcal{D}_{3} \times \mathcal{D}_{3}\right)$, where $\mathcal{S}_{\mathrm{CL}}$ and ${ }^{-}$are defined as in Ex. 1 . Note that $a=\top \Rightarrow p \in \mathcal{G}\left(\left(\operatorname{Arg}\left(A S_{2}\right)\right.\right.$, UrRe $\left.)\right)$ but since $b=\langle\top \Rightarrow s\rangle,\langle\top \Rightarrow \neg s\rangle \rightarrow \neg p \in \operatorname{Arg}\left(A S_{3}\right)$ and $b$ defeats $a, a \notin \mathcal{G}\left(\left(\operatorname{Arg}\left(A S_{3}\right), \mathrm{UrRe}\right)\right)$.

A solution to problems of this kind has been proposed in [Wu, 2012] for $\mathrm{ASPIC}^{+}$(so using restricted rebut) and for trivial preference orderings over the defeasible rules (so where $\leq=\mathcal{D} \times \mathcal{D}$ ). Non-Interference is ensured there by filtering out inconsistent arguments like $b$ in Ex. 2, i.e., arguments with inconsistent support sets.

A first proposal to ensure Non-Interference for $\sim^{\text {UrRe }}$ would be thus to filter out inconsistent arguments. However, in ASPIC $^{-}$frameworks with a non-trivial priority ordering this leads to violations of Closure as shown in the following example:

Example 3 (Ex. 1 continued). Suppose the inconsistent $a_{6}$ is removed from the $A S$ while the consistent arguments $a_{1}, a_{2}, a_{3}, a_{4}, a_{5}$ remain. Since $a_{5} \preceq a_{1}$ while $a_{1} \npreceq a_{5}$, with $a_{1}$ the argument $a_{5}$ can be defeated but not vice versa. Thus, $a_{1}$ is in the grounded extension $\mathcal{G}$ while $a_{5} \notin \mathcal{G}$. Moreover, the potential defeater $a_{6}$ of $a_{2}$ is removed, thus $a_{2} \in \mathcal{G}$. However, $a_{3}$ and $a_{4}$ keep each other out of the grounded extension. Hence, we get the consequences $\neg p \vee \neg q$ and $p$ while $\neg q$ does not follow.

\footnotetext{
${ }^{4}$ A related rationality standard is Crash Resistance. It follows from Non-Interference under some very weak criteria on the strict rule base (cf. [Caminada et al., 2011]).
}

We analyse this example as follows: $a_{1}=\top \Rightarrow_{2} \neg p \vee$ $\neg q$ offers a strong reason against the reasoning path taken by $a_{3}=\left\langle T \Rightarrow_{1} p\right\rangle \Rightarrow_{1} q$ since it expresses that at least one of the two conclusions of the two subarguments $a_{2}=\top \Rightarrow_{1} q$ and $a_{3}$ has to be false. In other words, someone arguing for $a_{3}$ is committed to both $p$ and $q$ while someone arguing for $a_{1}$ expresses that this commitment is mistaken. In view of this, one would expect $a_{1}$ to defeat $a_{3}$. Consequently, since $a_{1}$ is in the grounded extension, it would defend $a_{4}=a_{1}, a_{2} \Rightarrow_{1} \neg q$ from its attacker $a_{3}$. Thus, also $a_{4}$ would be grounded and we would get Closure.

Our analysis motivates to generalize Unrestricted Rebut to allow for attacks like the one of $a_{1}$ on $a_{3}$ as follows: $a$ attacks $b$ in (the conclusions of) some of its subarguments $b_{1}, \ldots, b_{n}$ iff $\operatorname{conc}(a)$ expresses that $\operatorname{conc}\left(b_{1}\right), \ldots, \operatorname{conc}\left(b_{n}\right)$ cannot all hold at the same time. We will do so in the next section.

\section{Generalizing Rebut: ASPIC ${ }^{\ominus}$}

We will now define ASPIC $^{\ominus}$, a framework for structured argumentation that satisfies all four rationality postulates above while allowing for unrestricted rebut. Recall that we faced the following dilemma in Section 4. Unrestricted rebut leads to a violation of Non-Interference (see Ex. 2). While removing inconsistent arguments from $\mathrm{AFs}$ remedies this shortcoming it gives rise to a violation of Closure (see Ex. 3). We will now show that we can have our cake and eat it: inspired by our analysis of Ex. 3 we propose a generalization of unrestricted rebut which allows for an argument to attack another one if its conclusion claims that a subset of the commitments of the attacked argument are not tenable together.

To formalize this idea we introduce two notational conventions. First, we assume that a lifting of the contrariness operator $^{-}: \mathcal{L} \rightarrow \mathcal{L}$ to (finite) sets of formulas ${ }^{-}: \wp_{\text {fin }}(\mathcal{L}) \backslash \emptyset \rightarrow \mathcal{L}$ is available. For instance, one may express $\overline{\left\{A_{1}, \ldots, A_{n}\right\}}$ by means of disjunction $\bigvee_{i=1}^{n} \overline{A_{i}}$ or by means of conjunction $\overline{\bigwedge_{i=1}^{n} A_{i}}$. Second, we define $\mathrm{C}(a)=_{\mathrm{df}}\{\operatorname{conc}(b) \mid b \in$ $\operatorname{Sub}(a)\}$ to be the set of the conclusions of subarguments of $a$.

Definition 9. Where $a, b \in \operatorname{Arg}(A S): a$ gen-rebuts $b$ (in symbols: $a \mathrm{GeRe} b)$ iff $b$ is defeasible and $\operatorname{conc}(a)=\bar{\Delta}$ for some $\Delta \subseteq \mathrm{C}(b)$.

Definition 10. $(a, b) \in \mathrm{GeRe}_{\preceq}$ iff there is $a c \in \operatorname{Sub}(b)$ s.t. $a \mathrm{GeRe} c$ and $c \preceq a$.

Clearly, if $a \mathrm{Ue} \operatorname{Re} b$ then $a \mathrm{GeRe} b$ while the other direction does not hold in general.

The consequence relation for generalized rebuttal $\sim^{\text {GeRe }}$ is defined as in Definition 8.

In Example 3, note that now $a_{1}$ defeats $a_{3}$ and consequently, $A S \sim^{\text {GeRe }} \neg p$. In fact, as is shown in the next section all four rationality postulates hold for $\sim^{\text {GeRe }}$ given some requirements on the strict rules that are met by many potential base logics or domain dependent rule bases.

\section{Rationality Standards and ASPIC ${ }^{\ominus}$}

When proving the rationality postulates for $\operatorname{ASPIC}^{\ominus}$, we will suppose that the set of strict rules $\mathcal{S}$ of a given AS satisfies 
Transposition $(\mathbf{T})$, Resolution $(\mathbf{R})$ and Cut $(\mathbf{C})$ (where $\Delta^{\prime} \subseteq$ $\Delta$ and $\Theta^{\prime} \subseteq \Theta$ are finite sets of formulas):

$$
\begin{aligned}
& \text { T: }\left(\Delta \backslash \Delta^{\prime}\right) \cup \Theta^{\prime} \rightarrow \overline{\left(\Theta \backslash \Theta^{\prime}\right) \cup \Delta^{\prime}} \text { if } \Delta \rightarrow \bar{\Theta} . \\
& \text { R: } \Delta \cup \overline{\Delta \cup \Theta} \rightarrow \bar{\Theta} . \\
& \text { C: } \Delta \cup \Theta \rightarrow A \text { if } \Delta \cup\{D\} \rightarrow A \text { and } \Theta \rightarrow D .
\end{aligned}
$$

We suppose that there is a conjunction symbol in the language that works in the usual way: e.g., $\Delta \rightarrow A$ iff $\bigwedge \Delta \rightarrow A$; $\Delta \rightarrow \bigwedge \Delta$ and $\bigwedge \Delta \rightarrow A$ where $A \in \Delta$ are available rules.

The generality of these requirements ensure that our framework can be instantiated by a broad class of rule bases. For example, a wide variety of Tarski consequence relations, such as $\mathbf{C L}$, intuitionistic logic and many modal logics, can be used to generate a set of strict rules. Likewise, closing a set of domain specific rules under the properties defined above generates such a strict rule base. Note that Transposition was already required in e.g. [Prakken, 2011] and [Caminada et al., 2014].

Example 4. For the instantiation $\mathcal{S}_{\mathbf{C L}}$ in terms of $\mathbf{C L}$ proposed in Ex. 1 the requirements read:

T: If $A_{1}, \ldots, A_{n} \rightarrow \neg B_{1} \vee \ldots \vee \neg B_{m} \in \mathcal{S}_{\mathbf{C L}}$ then $A_{1}, \ldots, A_{l}, B_{k}, \ldots, B_{m} \rightarrow \neg A_{l+1} \vee \ldots \vee \neg A_{n} \vee \neg B_{1} \vee$ $\ldots \vee \neg B_{k-1} \in \mathcal{S}_{\mathbf{C L}}$.

$R: A_{1}, \ldots, A_{n}, \neg A_{1} \vee \ldots \vee \neg A_{n} \vee \neg B_{1} \vee \ldots \vee \neg B_{m} \rightarrow$ $\neg B_{1} \vee \ldots \vee \neg B_{m} \in \mathcal{S}_{\mathbf{C L}}$.

C: If $A_{1}, \ldots, A_{n} \rightarrow A \in \mathcal{S}_{\mathbf{C L}}$ and $B_{1}, \ldots, B_{m} \rightarrow A_{i} \in \mathcal{S}_{\mathbf{C L}}$ then $A_{1}, \ldots, A_{i-1}, A_{i+1}, . ., A_{n}, B_{1}, . ., B_{m} \rightarrow A \in \mathcal{S}_{\mathbf{C L}}$.

As noted in Section 4, Non-Interference fails for unrestricted rebut in the presence of inconsistent arguments. In contrast, for generalized rebut they are harmless as the grounded extension attacks them:

Fact 1. Where $A S=\left(\mathcal{L}, \mathcal{S}, \mathcal{D}, \mathcal{K},{ }^{-}, \leq\right), \quad A F=$ $\left(\operatorname{Arg}(A S), \operatorname{GeRe}_{\preceq}\right), \mathcal{S}$ is closed under $\mathbf{T}$ and $\mathbf{C}$, and $b \in$ $\operatorname{Arg}(A S)$ is $\mathcal{S}$-inconsistent (i.e., $\mathrm{C}(b)$ is $\mathcal{S}$-inconsistent), b is defeated by $\langle\rightarrow \overline{\mathrm{C}(b)}\rangle \in \mathcal{G}_{0}(A F)$.

Proof. Since $b$ is inconsistent, $\exists A \in \mathcal{L}$ s.t. $\mathrm{C}(b) \rightarrow$ $A, \mathrm{C}(b) \rightarrow \bar{A} \in \mathcal{S}$. By $\mathbf{T}, A \rightarrow \overline{\mathrm{C}(b)} \in \mathcal{S}$. By $\mathbf{C}$, $\mathrm{C}(b) \rightarrow \overline{\mathrm{C}(b)} \in \mathcal{S}$. By $\mathbf{T}, a=\langle\rightarrow \overline{\mathrm{C}(b)}\rangle \in \operatorname{Arg}(A S)$. As $a$ has no attackers, $a \in \mathcal{G}_{0}(A F)$.

The four rationality standards hold for $\sim^{\text {GeRe }}$ :

Theorem 1. $\sim^{\text {GeRe }}$ satisfies Direct Consistency, Closure, Indirect Consistency and Non-Interference for any $A S=$ $\left(\mathcal{L}, \mathcal{S}, \mathcal{D}, \mathcal{K},{ }^{-}, \leq\right)$such that $\mathcal{S}$ is closed under $\mathbf{T}, \mathbf{R}$ and $\mathbf{C}$ and $\leq$ is a total preorder.

Proof. Direct Consistency follows from $\mathcal{G}(A F)$ being conflict-free. Closure follows from Lemma 2 (proven below). Indirect Consistency is a corollary of Direct Consistency and Closure. Non-Interference follows with Fact 5 and Lemmas 3 and 4 (proven below). We show one direction. Suppose $A S^{\prime} \sim^{\text {GeRe }} A$. Thus, $\exists a \in \mathcal{G}\left(A F^{\prime}\right)$ s.t. $\operatorname{conc}(a)=A$. By Fact 5, $\operatorname{cut}(a) \in \mathcal{G}\left(A F^{\prime}\right)$. By Lemma 3 , cut $(a) \in \mathcal{G}\left(A F^{+}\right)$. Thus $A S^{+} \sim^{\text {GeRe }} A$.
The following proofs are for a given AF with underlying argumentation system $A S=\left(\mathcal{L}, \mathcal{S}, \mathcal{D}, \mathcal{K}^{-}{ }^{-}, \leq\right)$that satisfies the requirements of Theorem 1 . In order to avoid clutter, we will in the following sometimes write $\overline{a_{1}, \ldots, a_{n}}$ instead of $\overline{\left\{\operatorname{conc}\left(a_{1}\right), \ldots, \operatorname{conc}\left(a_{n}\right)\right\}}$. Furthermore, we will say $a$ defeats $b$ in $\left\{b_{1}, \ldots, b_{n}\right\}$ if $a$ defeats $b$ and conc $(a)=\overline{b_{1}, \ldots, b_{n}}$ for $b_{1}, \ldots, b_{n} \in \operatorname{Sub}(b)$.

Some proofs of the following auxiliary results are omitted due to space restrictions:

Fact 2. $C_{1}, \ldots, C_{m} \rightarrow \overline{A_{1}, \ldots, A_{n}, B_{2}, \ldots, B_{k}} \in \mathcal{S}$ if $A_{1}, \ldots, A_{n} \rightarrow B_{1} \in \mathcal{S}$ and $C_{1}, \ldots, C_{m} \rightarrow \overline{B_{1}, \ldots, B_{k}} \in$ $\mathcal{S}$.

Lemma 1. If $a \in \mathcal{G}_{k}(A F)$ then $a^{\prime}=\langle a \rightarrow A\rangle \in \mathcal{G}_{k}(A F)$ where $k \geq 0$ and $\operatorname{conc}(a) \rightarrow A \in \mathcal{S}$.

Proof. We show the inductive step $(k \Rightarrow k+1)$ of the inductive proof. Suppose $b$ defeats $a^{\prime}$ in some $\Delta$. If $a^{\prime} \notin \Delta$ then $b$ defeats $a$ and is thus defeated by $\mathcal{G}_{k}$. Else by Fact 2 $b^{\prime}=\left\langle b \rightarrow \overline{(\Delta \cup\{a\}) \backslash\left\{a^{\prime}\right\}}\right\rangle$ defeats $a$. Thus, some $c \in \mathcal{G}_{k}$ defeats $b^{\prime}$ in some $\Lambda$. If $b^{\prime} \notin \Lambda, c^{\prime}$ also defeats $b$. Otherwise with Fact $2 c^{\prime}=\left\langle c \rightarrow \overline{(\Lambda \cup\{b\}) \backslash\left\{b^{\prime}\right\}}\right\rangle$ defeats $b$.

Fact 3. 1. If $b=\left\langle b_{1}, \ldots, b_{n} \rightarrow B\right\rangle$ and $\mathcal{G}_{k}$ defeats $b$ then it also defeats $b$ in some $\Delta$ for which $b \notin \Delta$.

2. If $a \in \mathcal{G}_{k}, b=\left\langle a_{1}, \ldots, a_{n} \rightarrow A\right\rangle \in \operatorname{Arg}(A S)$ where $a_{1}, \ldots, a_{n} \in \operatorname{Sub}(a)$, then $b \in \mathcal{G}_{k}$.

3. Where $a \in \mathcal{G}$ and a attacks $b$ then $\mathcal{G}$ defeats $b$.

Proof. Ad 1. Suppose $c \in \mathcal{G}_{k}$ defeats $b$ in $\Delta$ and $b \in \Delta$. By Fact 2 and Lemma $1 c^{\prime}=\left\langle c \rightarrow \overline{\left(\Delta \cup\left\{b_{1}, \ldots, b_{n}\right\}\right) \backslash\{b\}}\right\rangle \in$ $\mathcal{G}_{k}$. Clearly, $c^{\prime}$ defeats $b$. Ad 2. Suppose some $c$ defeats $b$ in $\Delta$. If $b \notin \Delta$ then $c$ also defeats $a$ and thus $G_{k-1}$ defeats $b$. Else by Fact $2 c^{\prime}=\left\langle c \rightarrow \overline{\left.\Delta \cup\left\{a_{1}, \ldots, a_{n}\right\}\right) \backslash\{b\}}\right\rangle$ defeats $a$. Again, $\mathcal{G}_{k-1}$ defeats $c^{\prime}$ and by item 1 it also defeats $b$. Ad 3. Omitted due to space.

Lemma 2. If $a_{1}, \ldots, a_{n} \in \mathcal{G}(A F)$ and $a=\left\langle a_{1}, \ldots, a_{n} \rightarrow\right.$ $A\rangle \in \operatorname{Arg}(A S), a \in \mathcal{G}(A F)$.

Proof. We prove this by an induction on $\pi(a)=n+$ $\sum_{i=1}^{n} \kappa\left(a_{i}\right)$ where $\kappa\left(a_{i}\right)$ is the minimal $j$ for which $a_{i} \in \mathcal{G}_{j}$. We show the inductive step. We first consider defeaters $b$ of $a$ in some $\Delta$ where $a \notin \Delta$. Let $\Delta_{i}=\Delta \cap \operatorname{Sub}\left(a_{i}\right)$ where $1 \leq i \leq n$. Suppose first that some $\Delta_{i}=\emptyset$. Wlog suppose $\Delta_{1} \cup \ldots \cup \Delta_{k}=\emptyset$ while each $\Delta_{j} \neq \emptyset$ where $k<j \leq n$. Let $d_{j}=\left\langle\Delta_{j} \rightarrow \bigwedge \Delta_{j}\right\rangle$. By Fact $3.2 d_{j} \in \mathcal{G}_{\kappa\left(a_{j}\right)}$. By the IH, $d^{\prime}=\left\langle d_{k+1}, \ldots, d_{n} \rightarrow \bar{c}\right\rangle \in \mathcal{G}$. Since $d^{\prime}$ attacks $c$, by Fact $3.3, \mathcal{G}$ defeats $c$.

Suppose now that for every $1 \leq i \leq n, \Delta_{i} \neq \emptyset$. Suppose wlog that $a_{1} \in \min _{\preceq}\left(\left\{a_{1}, \ldots, a_{n}\right\}\right)$. Thus, $c^{\prime}=$ $\left\langle c, \Delta_{2}, \ldots, \Delta_{n} \rightarrow \overline{\Delta_{1}}\right\rangle$ defeats $a_{1}$. Hence, there is a $e \in$ $\mathcal{G}_{\kappa\left(a_{1}\right)-1}$ that defeats $c^{\prime}$ in some $\Lambda$. By Fact 3.1 we can assume that $c^{\prime} \notin \Lambda$. Let $\Lambda_{j}=\Lambda \cap \operatorname{Sub}\left(a_{j}\right)$ for each $2 \leq j \leq n$ and $\Lambda_{c}=\Lambda \cap \operatorname{Sub}(c)$. Let $l_{j}=\left\langle\Lambda_{j} \rightarrow \Lambda \Lambda_{j}\right\rangle$ if $\Lambda_{j} \neq \emptyset$ and otherwise $l_{j}$ is the empty string. By Fact 3.2, $l_{j} \in \mathcal{G}_{\kappa\left(a_{j}\right)}$ whenever $\Lambda_{j} \neq \emptyset$. Let $e^{\prime}=\left\langle e, l_{2}, \ldots, l_{n} \rightarrow \overline{\Lambda_{c}}\right\rangle$. By the IH, $e^{\prime} \in \mathcal{G}$. Since $e^{\prime}$ attacks $c, \mathcal{G}$ defeats $c$ by Fact 3.3. 
Consider a $b$ defeating $a$ in $\Delta$ s.t. $A \in \Delta$. By Fact 2 we have $b^{\prime}=\left\langle b \rightarrow \overline{\left(\Delta \cup\left\{a_{1}, \ldots, a_{n}\right\}\right) \backslash\{a\}}\right\rangle$ defeating $a$. Above we have shown that $\mathcal{G}$ defeats $b^{\prime}$. By Fact $3.1 \mathcal{G}$ defeats $b$.

Remark 2. Any argument $a \in \operatorname{Arg}(A S)$ can be transformed into an argument cut $(a)$ such that: any argument $b \in \operatorname{Sub}(\operatorname{cut}(a))$ of the form $b=\left\langle b_{1}, \ldots, b_{n} \rightarrow B\right\rangle$ is such that each $b_{i}$ is either of the form $\left\langle B_{i}\right\rangle$ or of the form $\left\langle b_{1}^{\prime}, \ldots, b_{m}^{\prime} \Rightarrow B_{i}\right\rangle$, $\operatorname{DefR}(\operatorname{cut}(a))=\operatorname{DefR}(a)$, and $\mathrm{C}(\operatorname{cut}(a)) \subseteq \mathrm{C}(a)$.

The way to achieve this is to apply $\mathbf{C}$ "as much as possible". That means for any subargument $b=$ $\left\langle b_{1}, \ldots, b_{n} \rightarrow B\right\rangle$ of $a$ for which some $b_{i}$ is of the form $\left\langle b_{1}^{\prime}, \ldots, b_{m}^{\prime} \rightarrow B_{i}\right\rangle$ we replace $b$ in $a$ by $b^{\prime}=$ $\left\langle b_{1}, \ldots, b_{i-1}, b_{1}^{\prime}, \ldots, b_{m}^{\prime}, b_{i+1}, \ldots, b_{n} \rightarrow B\right\rangle$.

Fact 4. If $b$ is $\mathcal{S}$-consistent and $b^{\prime}=\langle b \rightarrow B\rangle \in \operatorname{Arg}(A S)$, then $b^{\prime}$ is $\mathcal{S}$-consistent.

Fact 5. If $a \in \mathcal{G}(A F)$ then also cut $(a) \in \mathcal{G}(A F)$.

In the remainder of this section, let $A S=\left(\mathcal{L}, \mathcal{S}, \mathcal{D}, \mathcal{K}{ }^{-}, \leq\right.$ ), $A S^{\prime}=\left(\mathcal{L}, \mathcal{S}, \mathcal{D}^{\prime}, \mathcal{K}^{\prime},{ }^{-}, \leq^{\prime}\right)$, and $A S^{+}=\left(\mathcal{S}, \mathcal{D} \cup \mathcal{D}^{\prime}, \mathcal{K} \cup\right.$ $\left.\mathcal{K}^{\prime},{ }^{-}, \leq^{+}\right)$be as in Postulate 4 where $A F, A F^{\prime}$ and $A F^{+}$are the corresponding argumentation frameworks.

Fact 6. Where $a \in \operatorname{Arg}\left(A S^{+}\right) \backslash \operatorname{Arg}\left(A S^{\prime}\right)$ is $\mathcal{S}$-consistent and $\operatorname{Atoms}(\operatorname{conc}(a)) \subseteq \operatorname{Atoms}\left(\mathcal{K}^{\prime} \cup \mathcal{D}^{\prime}\right)$, there is a $c \in \operatorname{Arg}\left(A S^{\prime}\right)$ for which $\mathrm{C}(c) \subseteq \mathrm{C}(a), \operatorname{DefR}(c)=\operatorname{DefR}(a) \cap \mathcal{D}^{\prime}$ and $\operatorname{conc}(c)=\operatorname{conc}(a)$.

Lemma 3. $\operatorname{cut}(a) \in \mathcal{G}\left(A F^{+}\right)$if $\operatorname{cut}(a) \in \mathcal{G}\left(A F^{\prime}\right)$.

Proof. Let $a^{\prime}=\operatorname{cut}(a)$. We prove by induction that if $a^{\prime} \in$ $\mathcal{G}_{i}\left(A F^{\prime}\right)$ then $a^{\prime} \in \mathcal{G}\left(A F^{+}\right)$. We show the inductive step. Let $a^{\prime} \in \mathcal{G}_{i+1}\left(A F^{\prime}\right)$. By Fact $1, a^{\prime}$ is $\mathcal{S}$-consistent. Suppose some $b \in \operatorname{Arg}\left(A S^{+}\right)$defeats $a^{\prime}$ in $\left\{a_{1}, \ldots, a_{n}\right\}$. If $b$ is $\mathcal{S}$ inconsistent it is, by Fact 1 , attacked by $\mathcal{G}_{0}\left(A F^{+}\right)$and hence $a^{\prime}$ is defended from $b$. Suppose now $b$ is $\mathcal{S}$-consistent.

If Atoms $\left(\operatorname{conc}\left(a^{\prime}\right)\right) \nsubseteq \nsubseteq \operatorname{Atoms}\left(A S^{\prime}\right)$ then $a^{\prime}$ is of the form $a^{\prime}=\left\langle c_{1}, \ldots, c_{m} \rightarrow A\right\rangle$. If now additionally $a^{\prime}=a_{i}$ for some $1 \leq i \leq n$, say wlog $a^{\prime}=a_{1}$, let $b^{\prime}=\langle b \rightarrow$ $\left.\overline{c_{1}, \ldots, c_{m}, a_{2}, \ldots, a_{n}}\right\rangle$ which is in $\operatorname{Arg}\left(A S^{+}\right)$by Fact 2 .

Else let $b^{\prime}=b$. By Fact $4, b^{\prime}$ is $\mathcal{S}$-consistent. By Fact 6, there is a $c \in \operatorname{Arg}\left(A S^{\prime}\right)$ s.t. $b^{\prime} \preceq^{\prime} c, \mathrm{C}(c) \subseteq \mathrm{C}(b)$, and $\operatorname{conc}(c)=\operatorname{conc}\left(b^{\prime}\right)$. Since $c$ defeats $a^{\prime}$ and $a^{\prime} \in$ $\mathcal{G}_{i+1}\left(A F^{\prime}\right)$ there is a $d \in \mathcal{G}_{i}\left(A F^{\prime}\right)$ that defeats $c$ in some $\left\{d_{1}, \ldots, d_{k}\right\}$. By the inductive hypothesis $d \in \mathcal{G}\left(A F^{+}\right)$. Since $\mathrm{C}(c) \subseteq \mathrm{C}\left(b^{\prime}\right), d$ also defeats $b^{\prime}$. If $\operatorname{conc}\left(b^{\prime}\right)$ is not among $\operatorname{conc}\left(d_{1}\right), \ldots, \operatorname{conc}\left(d_{k}\right), d$ defeats $b$ and hence defends $a$ from $b$. Otherwise suppose wlog, $\operatorname{conc}\left(d_{1}\right)=$ $\operatorname{conc}\left(b^{\prime}\right)$. By Fact $2, d^{\prime}=\left\langle d \rightarrow \overline{b, d_{2}, \ldots, d_{k}}\right\rangle \in \operatorname{Arg}\left(A S^{\prime}\right)$. By Lemma $1, d^{\prime} \in \mathcal{G}_{i}\left(A F^{\prime}\right)$. Since $d^{\prime}$ defeats $b$ it defends $a^{\prime}$. Altogether we have shown that $a^{\prime}$ is defended by $\mathcal{G}\left(A F^{+}\right)$ and thus $a^{\prime} \in \mathcal{G}\left(A F^{+}\right)$.

Lemma 4. Where Atoms $(\operatorname{conc}(a)) \subseteq \operatorname{Atoms}\left(\mathcal{D}^{\prime} \cup \mathcal{K}^{\prime}\right)$, if $a \in \mathcal{G}_{i}\left(A F^{+}\right)$then there is an $a^{\prime} \in \mathcal{G}_{i}\left(A F^{\prime}\right)$ with $\operatorname{conc}\left(a^{\prime}\right)=$ conc $(a), \mathrm{C}\left(a^{\prime}\right) \subseteq \mathrm{C}(a)$ and $\operatorname{DefR}\left(a^{\prime}\right)=\operatorname{DefR}(a) \cap \mathcal{D}^{\prime}$.

The proof is similar to the one of Lemma 3.

\section{Future Work}

In future research we plan to investigate how to overcome some of the restrictions imposed on the framework of this paper. This includes, among others, to study non-total orders on the priorities for the defeasible rules and other lifting principles such as last link, to combine generalized rebut with other attack rules such as undercut, and to study variants of generalized rebut. Generalized rebut in its current form allows for attacks that some may deem counter-intuitive. E.g., given the arguments $a=\left\langle\top \Rightarrow_{3} p\right\rangle \Rightarrow_{1} q$ and $b=\top \Rightarrow_{2} \neg p$, we have $b \operatorname{GeRe}_{\prec} a$ simply since $a \preceq b$. Of course, $c=\top \Rightarrow_{3} p$ defeats $b$ and defends $a$, as desired. Nevertheless, on intuitive grounds one may consider $b$ defeating $a$ as counter-intuitive since the subargument $c$ of $a$ (the conclusion of which $b$ attacks) is stronger than $b$. An alternative would thus be to define $a$ gen-rebuts $b$ in the conclusions of subarguments $b_{1}, \ldots, b_{n}$ of $b$ iff (I) $\operatorname{conc}(a)=\overline{\left\{\operatorname{conc}\left(b_{1}\right), \ldots, \operatorname{conc}\left(b_{n}\right)\right\}}$ and (II) $b_{i} \preceq a$ for all (or some) $1 \leq i \leq n$. We will investigate such variants in the future. This variant also turns out more promising when studying other lifting principles such as last link.

\section{References}

[Caminada and Amgoud, 2007] Martin Caminada and Leila Amgoud. On the evaluation of argumentation formalisms. Artificial Intelligence, 171(5):286-310, 2007.

[Caminada et al., 2011] Martin Caminada, Walter Carnielli, and Paul Dunne. Semi-stable semantics. Journal of Logic and Computation, page exr033, 2011.

[Caminada et al., 2014] Martin Caminada, Sanjay Modgil, and Nir Oren. Preferences and unrestricted rebut. In Computational Models of Argument (Proceedings of COMMA 14), pages 209-220. IOS Press, 2014.

[Dung, 1995] Phan Ming Dung. On the acceptability of arguments and its fundamental role in nonmonotonic reasoning, logic programming and n-person games. Artificial Intelligence, 77(2):321-357, 1995.

[Grooters and Prakken, 2016] Diana Grooters and Henry Prakken. Two aspects of relevance in structured argumentation: minimality and paraconsistency. Journal of Artificial Intelligence Research, 56:197-245, 2016.

[Modgil and Prakken, 2013] Sanjay Modgil and Henry Prakken. A general account of argumentation with preferences. Artificial Intelligence, 195:361-397, 2013.

[Modgil and Prakken, 2014] Sanjay Modgil and Henry Prakken. The ASPIC+ framework for structured argumentation: a tutorial. Argument \& Computation, 5(1):31-62, 2014.

[Prakken, 2011] Henry Prakken. An abstract framework for argumentation with structured arguments. Argument and Computation, 1(2):93-124, 2011.

[Wu, 2012] Yining Wu. Between Argument and Conclusion. Argument-based Approaches to Discussion, Inference and Uncertainty. $\mathrm{PhD}$ thesis, Universite Du Luxembourg, 2012. 